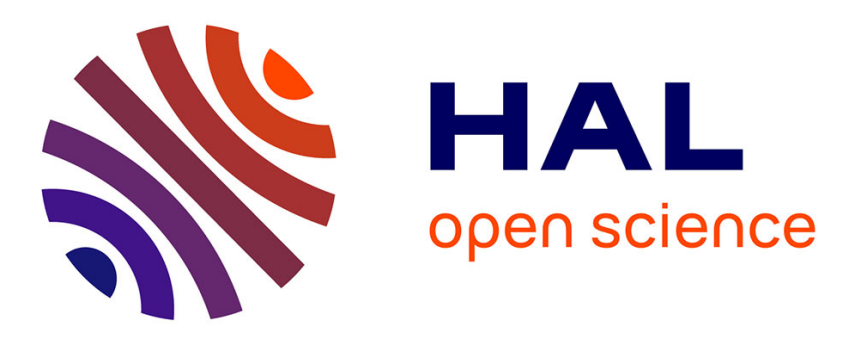

\title{
Excluded volume and hyperscaling in polymeric systems
}

\author{
M.E. Cates
}

\section{To cite this version:}

M.E. Cates. Excluded volume and hyperscaling in polymeric systems. Journal de Physique Lettres, 1985, 46 (17), pp.837-843. 10.1051/jphyslet:019850046017083700 . jpa-00232907

\section{HAL Id: jpa-00232907 https://hal.science/jpa-00232907}

Submitted on 1 Jan 1985

HAL is a multi-disciplinary open access archive for the deposit and dissemination of scientific research documents, whether they are published or not. The documents may come from teaching and research institutions in France or abroad, or from public or private research centers.
L'archive ouverte pluridisciplinaire HAL, est destinée au dépôt et à la diffusion de documents scientifiques de niveau recherche, publiés ou non, émanant des établissements d'enseignement et de recherche français ou étrangers, des laboratoires publics ou privés. 
Classification

Physics Abstracts

$05.50-61.40-64.60$

\title{
Excluded volume and hyperscaling in polymeric systems
}

\author{
M. E. Cates \\ Cavendish Laboratory, Madingley Road, Cambridge CB3 OHE, U.K.
}

(Reçu le 29 avril 1985, accepté sous forme définitive le 2 juillet 1985)

\begin{abstract}
Résumé. - On considère une distribution de masse moléculaire particulière, en loi de puissance, $n(m) \sim m^{-\tau}$ (avec coupure pour les petites et les grandes valeurs) pour des chaînes polymères s'évitant les unes les autres dans un fondu, à $d$ dimensions. On propose que, pour $\tau$, il existe un domaine spécial, $d / d_{\mathrm{fg}}<\tau-1<d / d_{\mathrm{fs}}, d_{\mathrm{fg}}$ étant la dimension fractale d'une chaîne Gaussienne et $d_{\mathrm{fs}}$ celle d'une chaîne complètement gonflée. Dans ce domaine de $\tau$, les chaînes ne sont ni Gaussiennes ni gonflées, mais leur dimension fractale obéit à une relation d'" hyperscaling », $d_{\mathrm{f}}=d /(\tau-1)$. Ce résultat s'applique aux polymères linéaires $\left(d_{\mathrm{fg}}=2, d_{\mathrm{fs}}=1 / v(d)\right)$ et aux fractaux de polymères de connectivité quelconque. On dérive également les lois d'échelle dans le cas de solutions.
\end{abstract}

\begin{abstract}
A dense system of mutually avoiding polymers, obeying a quenched power-law mass distribution, $n(m) \sim m^{-\tau}$ (with low and high cutoffs) is considered in $d$ dimensions. It is argued that, for a certain range of $\tau\left(d / d_{\mathrm{fg}}<\tau-1<d / d_{\mathrm{fs}}\right)$, the chains are neither Gaussian (fractal dimension $\left.d_{\mathrm{f}}=\mathrm{d}_{\mathrm{fg}}\right)$ nor fully swollen $\left(d_{\mathrm{f}}=d_{\mathrm{fs}}\right)$, but instead obey exactly a hyperscaling relation, $d_{\mathrm{f}}=d /(\tau-1)$. This result applies both to linear polymers $\left(d_{\mathrm{fg}}=2, d_{\mathrm{fs}}=1 / v(d)\right)$ and to " polymeric fractals" (e.g., sol-molecules) of general connectivity. Scaling laws for dilution are also given.
\end{abstract}

\section{Introduction.}

The excluded volume problem, for polymers in athermal solvents and in melts, has been studied for many years $[1,2]$. To a large extent, the behaviour of polymers in these systems can be described by a small number of universal scaling functions and exponents. One of the most important exponents is $d_{\mathrm{f}}$, the fractal dimension, which governs the scaling of the radius, $R(m)$, of a chain with its mass, $m: R(m) \sim m^{1 / d_{\mathrm{f}}}$. It is known that, in a dilute athermal solution of linear polymers, the excluded volume interaction causes chains to be swollen, with $d_{\mathrm{f}}=d_{\mathrm{fs}}=1 / v(d)$, where $v(d) \simeq(d+2) / 3$ for $d<4$ [1]. Under melt conditions, however, the excluded volume interaction is screened, and the chains are Gaussian $\left(d_{\mathrm{f}}=d_{\mathrm{fg}}=2\right)$. The semi-dilute regime, in which chains are overlapping but not dense, is understood in terms of a crossover from swollen to Gaussian behaviour on a certain length scale [1].

It has been pointed out before [3] that intermediate values of $d_{\mathrm{f}}$ might be expected in the presence of a power-law distribution of chain lengths (masses), $n(m) \sim m^{-\tau}$. Previous attempts to calculate this effect directly [3] have been at the level of Flory theory, with the incorporation of a mass- 
scale dependent screening factor, obtained by mean field arguments $\left({ }^{1}\right)$. Very recently, however, Petschek, Pfeuty and Wheeler [4] have studied a family of systems with (discrete) values of $\tau$ lying in the range $d / d_{\mathrm{fg}}<\tau-1 \leqslant d / d_{\mathrm{fs}}$, and obtained by scaling arguments the generic result $d_{\mathrm{f}}=d /(\tau-1)$. Their calculation is possible because of a mapping between these special systems, which contain linear and ring polymers, and the $\mathrm{O}(n)$ model of magnetism with integer values of $n \geqslant 0$. The result $d_{\mathrm{f}}=d /(\tau-1)$ then corresponds to the hyperscaling relation. The case $n=1$ is expected to describe the equilibrium polymerization of sulphur $[4,5]$.

In this Letter I suggest a powerful direct approach to the excluded volume problem for general polymeric systems with power-law polydispersity. Using this approach I obtain results, which I argue to be exact, for $d_{\mathrm{f}}$ (in terms of $d_{\mathrm{fg}}$ and $d_{\mathrm{fs}}$ ) over the whole range of $\tau$. The main arguments are heuristic, but they are underpinned by a more rigorous treatment, based on perturbation theory, which is given in outline (in Sect. 3).

We will see that for $\tau<1+d / d_{\mathrm{fg}}$ the polymers are Gaussian $\left(d_{\mathrm{f}}=d_{\mathrm{fg}}\right)$, and for $\tau>1+$ $d / d_{\mathrm{fs}}$, they are fully swollen $\left(d_{\mathrm{f}}=d_{\mathrm{fs}}\right)$. In contrast, for $d / d_{\mathrm{fg}}<\tau-1<d / d_{\mathrm{fs}}$, the above-mentioned result, $d_{\mathrm{f}}=d /(\tau-1)$, is obtained quite generally. The analysis has, moreover, a simple and very powerful generalization to the case of a power-law mass distribution of " polymeric fractals " [6] of arbitrary (self-similar) connectivity. This covers experimentally important examples of polymer sols, which arise by a wide variety of polymerization and gelation processes [7-9]. In the special case of equilibrium percolation, the result again maps onto the hyperscaling relation (in the Potts model formulation [7]). However, it must be stressed that the present treatment relies only on the presence of excluded volume interactions between flexible polymeric clusters. It is therefore applicable to many systems, particularly those arising by irreversible polymerization processes, in which a power-law mass distribution of (linear or fractally connected) polymers is expected [8,9], but for which no mapping onto a magnetic system is available. Some implications for studies of these processes are discussed in the final sections of the Letter.

We start in section 2 , however, by considering the simplest case : a dense melt of linear polymers. The effects of dilution, and the extension of the theory to cover polymeric fractals, are discussed below (in Sects. 4 and 5).

\section{Polydisperse melts.}

Consider a set of linear polymers, each of which is embedded as a chain of $m$ sites on a lattice of unit bond-length, in $d$ dimensions. The number density of chains of " mass " $m$ is taken to be $n(m)=m^{-\tau} f\left(m / m_{0}, m / M\right)$, where $M \gg m_{0} \gtrsim 1$. For definiteness, we take $f(a, b)$ to be a constant, $\kappa$, for $b<1<a$, and zero otherwise. The chains take with equal probability all configurations in which no site is multiply occupied. The total chain concentration (fraction of occupied sites), $c$, is given by $c \simeq \int n(m) m \mathrm{~d} m=1\left({ }^{2}\right)$.

In studying the excluded volume problem for this dense system of chains, the crucial question centres on the presence or absence of screening effects. Essentially, if chains " overlap ", the excluded volume (e.v.) interaction between one chain and itself is screened by that between the chain and its neighbours. However in the polydisperse melt here considered, the definition of overlap is more subtle than one might expect. First, let us suppose the chains to have some

$\left({ }^{1}\right)$ We will see that such arguments miss a central feature of the problem, which is a sudden change in the quality of chain overlap (and an associated breakdown in mean field type averaging) at some ( $\tau$-dependent) value of $d_{\mathrm{f}}$.

${ }^{(2)}$ For $\tau<2, c \propto \kappa M^{2-\tau}$, whereas, for $\tau>2, c \propto \kappa m_{0}^{2-\tau}$. This means that for $\tau>2$ one can take the limit $M \rightarrow \infty$ at fixed $\kappa$ without violating the packing constraint $c \leqslant 1$. 
« imposed » fractal dimension, $d_{\mathrm{f}}$ (the equilibrium value of which we would like to find). A suitable (scale-dependent) measure of the overlap is then provided by $\Phi(m)$, the total hard sphere volume fraction (h.s.v.f.), of chains larger than a certain mass, $m(\ll M)$. The hard-sphere volume of a chain of mass $m$ varies as $R(m)^{d} \sim m^{d / d_{f}}$. We therefore define

$$
\Phi(m) \sim \int_{m} n\left(m^{\prime}\right) R\left(m^{\prime}\right)^{d} \mathrm{~d} m^{\prime} .
$$

It is important to consider carefully the dependence of $\Phi$ on the mass scale, $m$. We find from (1)

$$
\Phi(m) \sim \kappa\left|M^{1-\tau+d / d_{\mathrm{f}}}-m^{1-\tau+d / d_{\mathrm{f}}}\right| .
$$

Thus if $\tau<1+d / d_{\mathrm{f}}, \Phi(m) \rightarrow \infty$ as $M \rightarrow \infty\left({ }^{2}\right)$, and $\Phi(m) \gg 1$ for any $m$ (if $M$ is sufficiently large). Conversely, if $\tau>1+d / d_{\mathrm{f}}, \Phi(m) \rightarrow 0$ for $m$ large (but still $\ll M$ ). These remarks allow us to identify, for dense chains of fixed $d_{\mathrm{f}}$, the following three qualitatively distinct regimes of $\tau$. (i) When $\tau<1+d / d_{\mathrm{f}}$, the chains are " quasi-concentrated " in the sense that any chain is strongly overlapping with others of a size comparable with, or larger than itself. (ii) When $\tau>$ $1+d / d_{\mathrm{f}}$, the chains are " quasi-dilute ". This means that even though the many-chain system is space-filling $(c=1)$, any given chain of mass $m \gg 1$ has negligible overlap with molecules of a comparable or larger size. Instead it is entirely surrounded by chains very much smaller than itself. (iii) Thirdly, when $\tau=1+d / d_{\mathrm{f}}$, we have a " quasi- $c^{*}$ " regime, in which the h.s.v.f. of all chains adds up to roughly one $\left({ }^{3}\right)$. The chains are then marginally overlapped on all length scales $\left({ }^{4}\right)$.

2.1 SCREENING. - The next step is to consider the nature of screening of the excluded volume interaction in these three regimes of $\tau$. (For the moment, we still imagine $d_{\mathrm{f}}$ to be fixed at some arbitrary value). We start with the quasi-concentrated regime. First, note that connectivity on a length scale much larger than the size of some given molecule does not help to screen its e.v. interactions [10]. To account for this, we define a modified overlap parameter,

$$
\tilde{\Phi}(m) \sim R(m)^{d} m^{-1} \int_{m} n\left(m^{\prime}\right) m^{\prime} \mathrm{d} m^{\prime} .
$$

That is, we imagine cutting all larger chains into pieces of mass $m$ and work out the total h.s.v.f., $\tilde{\Phi}(m)$, of the new system. However, $\tilde{\Phi}(m)$ differs from $\Phi(m)$ by at most a numerical factor $\left({ }^{3}\right)$. Thus, as $M \rightarrow \infty, \tilde{\Phi}(m) \rightarrow \infty$ for any $m$; accordingly one expects e.v. effects to be screened very efficiently.

In the quasi-dilute regime, however, there is no screening of the e.v. interaction for any chain with $m \gg 1$. This is because connectivity on a length scale much smaller than the size of a given molecule also does not help screen the e.v. interaction between points on that molecule. More precisely, for a chain of mass $\boldsymbol{m}^{\prime}$ to contribute effectively to the screening of e.v. between points on a larger chain of mass $m, m^{\prime}$ must at least be of order $m^{x}$, where $x$ is strictly positive. (In Flory Huggins theory, for example, $x=1 / 2$ [10]). However, a chain of mass $m \gg 1$ has no overlap with any such chain : $\Phi\left(m^{\prime}=m^{x}\right) \rightarrow 0$ as $m$ becomes large, for any $x>0$. Thus (whatever the precise value of $x$ ) there can be no screening for large chains. Finally, in the quasi- $c^{*}$ regime, we expect the screening effect to be influenced in a complicated way by the marginal onset of chain overlap.

$\left({ }^{3}\right)$ Note that at the quasi- $c^{*}$ point, $\Phi(m) \sim \ln (M / m)$ but $\tilde{\Phi}(m)$ (see Eq. (3)) is a pure number.

$\left({ }^{4}\right)$ Note that the effect of dilution (see Eqs. $(6,7)$ below) is to alter only the $\kappa$-dependence in equation (2). This means that, even for $c \ll 1$, as $M \rightarrow \infty$, all these remarks still apply to large enough chains. 
2.2 Chain swelling. - Above we have imagined $d_{\mathrm{f}}$ to be fixed and considered the nature of the excluded volume interactions. In fact $d_{\mathrm{f}}$ is not fixed, but itself depends on whether or not screening is present (and hence on $\tau$ ).

For $\tau<1+d / d_{\mathrm{fg}}$, the chains are Gaussian and strongly overlapping. The e.v. interaction is screened and so this result is self-consistent. Similarly, for $\tau>1+d / d_{\mathrm{fs}}$, the chains are fully swollen, and quasi-dilute. There is no screening, and again this is self-consistent. However, between these two regimes there lies an interval, $1+d / d_{\mathrm{fg}}<\tau<1+d / d_{\mathrm{fs}}$, in which neither picture is adequate. If the chains are Gaussian, they do not overlap and should therefore swell. If they are swollen, they strongly interpenetrate, and the driving force for chain swelling is, paradoxically, absent.

Suppose then, we have a dense system of chains (with $\tau$ in this interval) which are unconstrained (no e.v. interaction) and therefore Gaussian. I now argue that if the interaction is switched on, the chains swell on all length scales until they reach a fractal dimension $d_{\mathrm{f}}$ exactly obeying the marginal overlap (quasi- $c^{*}$ ) criterion, $d_{\mathrm{f}}(\tau-1)=d$. At this point, the driving force for further swelling ceases abruptly, because any further change in $d_{\mathrm{f}}$ would lead to effectively complete screening.

This behaviour is somewhat reminiscent of a monodisperse polymer solution near the overlap threshold, $c^{*}$, when for some values of $c$, Gaussian chains do not interpenetrate, but swollen ones do. In the monodisperse case, the system resolves itself by finding the semi-dilute regime, in which chains are swollen up to some length scale (the blob size) but Gaussian at larger scales. In the polydisperse melt considered here, that is impossible; there can be no new relevant length, because chains of all sizes are faced with the same overlap paradox on every length scale. Instead, $d_{\mathrm{f}}$ itself has to change.

To summarize, I predict the following dependence of $d_{\mathrm{f}}$ on $\tau$ :

$$
\begin{array}{ll}
d_{\mathrm{f}}=d_{\mathrm{fg}} & \left(\tau<1+d / d_{\mathrm{fg}}\right) \\
d_{\mathrm{f}}=d /(\tau-1) & \left(1+d / d_{\mathrm{fg}}<\tau<1+d / d_{\mathrm{fs}}\right) \\
d_{\mathrm{f}}=d_{\mathrm{fs}} & \left(1+d / d_{\mathrm{fs}}<\tau\right)
\end{array}
$$

For systems of linear (or ring) polymers in $d=3, d_{\mathrm{f}}$ lies intermediate between $d_{\mathrm{fg}}=2$ and $d_{\mathrm{fs}} \simeq 5 / 3$ whenever $5 / 2<\tau \lesssim 2.8$. The $\mathrm{O}(n)$ models of reference [4] provide definitive examples of systems obeying this general result.

The results (4) for polydisperse melts should apply to all chains which are sufficiently long. If the shortest chains present are not monomers but themselves long chains $\left(m_{0} \gg 1\right)$, we expect there to be a crossover from Gaussian behaviour at short scales to partly or fully swollen behaviour (as described by (4b) or (4c)) at larger ones. Mean field arguments suggest that the crossover mass, $m_{\mathrm{c}}$, obeys $m_{\mathrm{c}} \simeq m_{0}^{2}$ [10]. However, so long as $m_{0}$ is not large but of order unity, $m_{\mathrm{c}}$ is also small, and (4b) or (4c) is obeyed by all chains of mass $m \gg 1$.

\section{Perturbation theory.}

A more rigorous justification of the results (4) can be given in the context of perturbation theory. That screening is complete in a quasi-concentrated system, may be shown by a method following that of Edwards [11]. One studies the many chain system, but fixes a single test chain to have $d_{\mathrm{f}}=d_{\mathrm{f} 1}$ whilst all other chains have some $d_{\mathrm{f}}=d_{\mathrm{f} 2}$ chosen so that $d_{\mathrm{f} 2}<d /(\tau-1)$. One then switches on the e.v. interaction between all chains, and examines, perturbatively, the effect this has on $d_{\mathrm{f} 1}$. By resummation of screening diagrams, and then power counting, one can show that (after resummation) the interaction between points on the test chain results in a perturbation to $d_{\mathrm{f} 1}$ which is irrelevant to all orders in the theory unless $d_{\mathrm{f} 1}$ strictly exceeds $d_{\mathrm{f} 2}$ by an amount proportional to $\left[d /(\tau-1)-d_{\mathrm{f} 2}\right]$. Finally requiring that $d_{\mathrm{f} 1}=d_{\mathrm{f} 2}=d_{\mathrm{f}}$ in equilibrium leads to the 
conclusion that values of $d_{\mathrm{f}}<d /(\tau-1)$ cannot occur unless $\tau<1+d / d_{\mathrm{fg}}$, when instead $d_{\mathrm{f}}=d_{\mathrm{fg}}$.

Likewise, for the quasi-dilute regime one can follow des Cloizeaux [12] and sum terms contributing to the second virial coefficient between chains. Arguing that this resummed interaction corresponds to a (scale dependent) hard-sphere repulsion, we find, by power counting, that for $d_{\mathrm{f}}>d /(\tau-1)$ the net interaction between different chains is now irrelevant. Thus, as previously stated, there can be no screening in this regime, and $d_{\mathrm{f}}>d /(\tau-1)$ is only possible if $d_{\mathrm{f}}=d_{\mathrm{fs}}$. Combining these results, we obtain equations (4a-c).

\section{Dilution.}

If $c=1$, the above picture is essentially complete; the chains are fractals with dimension $d_{\mathrm{f}}$ given by equations (4) on all length scales larger than the lattice spacing. (This applies so long as the low mass cutoff $m_{0}$ is itself of order unity, which we from now on assume). If we dilute the system, the behaviour is altered, but only at short length scales. This is reflected by the presence of a blob size [1], $\xi_{b}$, using which we can, by a simple re-scaling (taking a new lattice spacing at $\xi_{b}$ ) convert the dilute system into an effective $c=1$ system, to which the previous analysis applies. For $\tau<2, \xi_{\mathrm{b}}$ obeys $\xi_{\mathrm{b}}^{d_{\mathrm{s}}-d} \sim c$. This is the usual result $[1,13]$, arising from the requirement that the blobs are both fully swollen and space-filling. However, for $\tau>2$, the main contribution to $c$ is from very short chains. Any chain much smaller than a blob cannot contribute to the " mesh " of which $\xi_{b}$ is the correlation length. Following through this argument leads to a new $\left({ }^{5}\right)$, selfconsistent result

$$
\xi_{\mathrm{b}}^{d_{\mathrm{fs}}(\tau-1)-d} \propto \kappa(\tau>2)
$$

which applies so long as $\tau<1+d / d_{\mathrm{fs}}$. (We recall that if $\tau>1+d / d_{\mathrm{fs}}$, the chains are fully swollen even under dense conditions, and dilution therefore has no further effect on their statistics). Using equation (5), we find for $2<\tau<1+d / d_{\mathrm{fg}}$,

$$
R(m) \sim m^{1 / d_{\mathrm{fg}}} \kappa^{\alpha},
$$

with $\alpha=\left[d_{\mathrm{fs}} / d_{\mathrm{fg}}-1\right] /\left[d_{\mathrm{fs}}(1-\tau)+d\right]$. On the other hand, for chains obeying the marginal overlap criterion (Eq. (4b)),

$$
R(m) \sim m^{1 / d_{\mathrm{f}}} \kappa^{-1 / d} .
$$

These results apply when $m \gg m_{\mathrm{b}}=\xi_{\mathrm{b}}^{d}$. Substituting the result (7) in equation (3), we find that the effective overlap parameter in the quasi- $c^{*}$ regime, $\tilde{\Phi}(m)$ is a constant $(\phi$, say) independent not only of $m_{0}, m$ and $M$ but also of $\kappa$. All the complicated effects associated with the breakdown of screening at the quasi- $c^{*}$ point are reflected not in $d_{\mathrm{f}}$ (which is fixed already by the marginal overlap requirement) but in the dimensionless number $\phi$.

\section{Extension to polymeric fractals.}

Nowhere in the above calculation has explicit use been made of the linear connectivity of the polymers under study; this only enters through the values of $d_{\mathrm{fg}}$ and $d_{\mathrm{fs}}$. Suppose then that we had a collection of polymeric fractals [6] of some more complex (but self-similar) connectivity, or more generally obeying a (quenched) distribution of such connectivities, in addition to a quenched power-law distribution of masses characterized by $\tau$. In this case, $d_{\mathrm{fg}}$ and $d_{\mathrm{fs}}$ will be

$\left({ }^{5}\right)$ The fact that very small chains cannot contribute to the mesh leads to results which differ from those of, e.g., reference [13]. 
replaced by new values, but, so long as these quantities are well defined as ensemble properties, the entire analysis leading to equations (1-7) (including, in fact, the perturbation theory) carries over unaltered subject to this replacement. In general, $d_{\mathrm{fg}}$ is given by $d_{\mathrm{fg}}=\inf \left[2 d_{\mathrm{s}} /\left(2-d_{\mathrm{s}}\right), d\right]\left(^{6}\right)$ where $d_{\mathrm{s}}<2$ is the spectral dimension [14] of the polymers. The value of $d_{\mathrm{fs}}$ will in general depend, not only on $d_{\mathrm{s}}$, but on other parameters describing detailed aspects of the polymer connectivity. However, at the level of Flory theory, these details do not matter, and the fully swollen fractal dimension, $d_{\mathrm{fs}}$, is given by $d_{\mathrm{fs}} \simeq d_{\mathrm{s}}(d+2) /\left(d_{\mathrm{s}}+2\right)[6]$.

5.1 IMPLICATIONS FOR THEORIES OF GELATION. - The percolation model of gelation defines, with certain weights, a set of mutually avoiding clusters on a lattice. It is known that for percolation in dimension $d>6, d_{\mathrm{s}}=4 / 3, d_{\mathrm{f}}=d_{\mathrm{fg}}=4$ and $\tau=5 / 2$. For $d<6, d_{\mathrm{f}}$ and $\tau$ are not calculable exactly, but obey $d_{\mathrm{f}}=d /(\tau-1)$, which corresponds to the hyperscaling relation in a Potts model formulation [7].

These familiar results can now be given a new interpretation purely in terms of excluded volume. We restrict attention to the sol phase, and imagine the distribution of cluster masses and connectivities (but not spatial arrangements) to be fixed. We now try to calculate $d_{\mathrm{f}}$ in terms of parameters of this distribution (such as $\tau$ and $d_{\mathrm{s}}$ ). Since bond rotations are allowed, the clusters are completely flexible at large scales [15], and hence [6] we may treat them as a set of mutually avoiding polymeric fractals. We see that for $d>6, \tau<1+d / d_{\mathrm{fg}}$, and so, by equation (4a), $d_{\mathrm{f}}=d_{\mathrm{fg}}=4$ as expected. For $d<6$, on the other hand, the hyperscaling result, $d_{\mathrm{f}}=d /(\tau-1)$, is obtained so long as the exponents $d_{\mathrm{fg}}, d_{\mathrm{fs}}$ and $\tau$ obey $d / d_{\mathrm{fg}}<\tau-1<d / d_{\mathrm{fs}}$. This requirement (which is actually rather weak) seems to be safely obeyed by the best available estimates of these exponents in $2 \leqslant d \leqslant 6\left({ }^{7}\right)$. Thus I argue that the hyperscaling result can be understood purely in terms of two-body excluded volume forces between flexible clusters. This is not obvious from the Potts model formulation [7], nor from previous « polymeric » formulations $[3,7,13]$ of the percolation model.

The result is important, because the same reasoning can now be applied to polymer sols arising by mechanisms other than equilibrium percolation. A number of models of such processes have been suggested recently [7-9, 16]. In general, one expects the exponents for these processes to differ from those of the percolation model. However, if one assumes equilibrium with respect to excluded volume forces (and in, say, a quenched polymer sol, this should presumably be true for all realistic models) then the arguments given above suggest that hyperscaling, in the form $d_{\mathrm{f}}=d /(\tau-1)$, may be obeyed under much broader conditions than have previously been suspected. For this to apply in any particular case, the polymer exponents, $d_{\mathrm{fg}}, d_{\mathrm{fs}}$, and $\tau$ do not (as one might naively expect) have to obey some special equality. Instead, only a relatively weak pair of inequalities (Eq. (4b)) need be obeyed.

\section{Conclusions.}

The hyperscaling relation, $d_{\mathrm{f}}=d /(\tau-1)$, is expected to arise in polymeric systems under relatively broad conditions, described by equation (4b) above. Explicit examples are provided by the $\mathrm{O}(n)$ models of equilibrium polymerization (of linear and ring polymers) of reference [4], and also by the percolation model (when viewed as a system of polymeric fractals). The result can, however, be understood purely in terms of a two-body excluded volume interaction between flexible clusters; the derivation does not require the use of a mapping onto a magnetic analogue.

$\left({ }^{6}\right)$ Whenever ideal Gaussian polymeric fractals have $d_{\mathrm{fg}}=2 d_{\mathrm{s}} /\left(2-d_{\mathrm{s}}\right)>d$ (see Ref. [6]), real (screened) ones have $d_{\mathrm{fg}}=d$ (i.e., a saturating density).

${ }^{7}$ ) Estimates of $\tau$ are from reference [7] and references therein; estimates of $d_{\mathrm{fg}}$ and $d_{\mathrm{fs}}$ are found, e.g., from those for $d_{\mathrm{s}}$ of reference [14], by using the results of reference [6]. 
An important corollary is that hyperscaling may prove to be commonplace in systems, such as those formed by irreversible polymerization, for which no such analogue exists.

Direct application of scaling arguments leads ta the dilution laws (5) to (7). For systems obeying hyperscaling, the effective overlap parameter $\tilde{\Phi}(m)=\phi$, turns out to be independent, not only of the mass scale $(m)$, but also of the parameter $\kappa$ which governs the total chain concentration. It would be interesting to see whether, in systems with a magnetic analogue, $\phi$ can be related to a critical amplitude.

\section{Acknowledgments.}

I wish to thank Dr R. C. Ball for several valuable suggestions, and also Prof. S. F. Edwards for helpful discussions. The financial support of the Science and Engineering Research Council (U.K.) and Esso Petroleum Ltd. is gratefully acknowledged.

\section{References}

[1] De Gennes, P. G., Scaling Concepts in Polymer Physics (Cornell Univ. Press, Ithaca) 1979, and references therein.

[2] Flory, P., Principles of Polymer Chemistry (Cornell Univ. Press, Ithaca) 1971.

[3] IsAacson, J. and Lubensky, T. C., J. Physique Lett. 41 (1980) L-469 ;

De Gennes, P. G., C. R. Acad. Sci. Paris B 291 (1980) 17.

[4] Petschek, R. G., Pfeuty, P. and Wheeler, J. C., J. Physique Lett. 45 (1984) L-1183. In the notation of these authors, $\tau(n)=1+[2-\alpha(n)] / \phi(n)$.

[5] CoRdery, R., Phys. Rev. Lett. 47 (1981) 457;

PFeuty, P. and Wheeler, J. C., Phys. Lett. A 84 (1981) 493.

[6] Cates, M. E., Phys. Rev. Lett. 53 (1984) 926 ;

CATES, M. E., J. Physique (1985) in press.

Polymeric fractals are flexible polymer networks of self similar connectivity on scales much larger than a constituent " bond ». Each bond itself consists of a long, fully flexible, piece of linear chain.

[7] For a review, see Stauffer, D., Coniglio, A. and Adam, M., in Advances in Polymer Science 44, 103-158, Ed. K. Dusek (Springer, Berlin) 1982.

[8] ZifF, R. M., J. Stat. Phys. 23 (1980) 241 ;

Ziff, R. M., Hendriks, E. M. and ERnst, M. H., Phys. Rev. Lett. 49 (1982) 593 ;

ERnst, M. H., Hendriks, E. M. and Leyvraz, F., J. Phys. A 17 (1984) 2137.

[9] Manneville, P. and De Seze, L., in Numerical Methods in the Study of Critical Phenomena, Eds. I. Della Dora, J. Demangeot and B. Lacolle (Springer-Verlag, Berlin) 1981 ;

Hermann, H. J., Stauffer, D. and Landau, D. P., J. Phys. A 16 (1983) 1221.

[10] Flory, P., J. Chem. Phys. 17 (1949) 303 ;

Joanny, J. F., Grant, P., Turkevich, L. and Pincus, P., J. Physique 42 (1981) 1045 ; see also Ref. [1] p. $59-60$.

[11] Edwards, S. F., Proc. Phys. Soc. London 88 (1966) 265 ; see also Ref. [1] p. 85-88.

[12] Des Cloizeaux, J., J. Physique 42 (1981) 635-652.

[13] Alexander, S., Grest, G. S., Nakanishi, H. and Witten, T. A., J. Phys. A 17 (1984) L185

[14] AlÉXAnder, S. and OrbaCh, R., J. Physique Lett. 43 (1982) L-625.

[15] see, e.g., Kantor, Y. and Webman, I., Phys. Rev. Lett. 52 (1984) 1891.

[16] Alexandrowicz, Z., Phys. Rev. Lett. 54 (1985) 1420 ; Proceedings of the International Topical Conference on the Kinetics of Aggregation and Gelation, Eds. F. Family and D. P. Landau (North Holland, Amsterdam) 1984. 